\title{
Astrochemistry: From the Chemical Laboratory to the Stars Virtual Issue
}

Tunneling Reaction Kinetics for the Hydrogen Abstraction Reaction $\mathrm{H}+\mathrm{H}_{2} \mathrm{~S} \rightarrow \mathrm{H}_{2}+\mathrm{HS}$ in the Interstellar Medium

Thanja Lamberts and Johannes Kästner

The Journal of Physical Chemistry A 2017, 121 (51), 9736-9741

DOI: $\underline{10.1021 / a c s . j p c a .7 b 10296}$

Variationally Computed IR Line List for the Methyl Radical $\mathrm{CH}_{3}$

Ahmad Y. Adam, Andrey Yachmenev, Sergei N. Yurchenko, and Per Jensen

The Journal of Physical Chemistry A 2019, 123 (22), 4755-4763

DOI: $10.1021 /$ acs.jpca.9b02919

Excited State Trends in Bidirectionally Expanded Closed-Shell PAH and PANH Anions

Ryan C. Fortenberry, Megan M. Moore, and Timothy J. Lee

The Journal of Physical Chemistry A 2016120 (37), 7327-7334

DOI: $10.1021 / a c s . j p c a .6 b 06654$

Tunneling Isomerizations on the Potential Energy Surfaces of Formaldehyde and Methanol Radical Cations

J. Philipp Wagner, Marcus A. Bartlett, Wesley D. Allen, and Michael A. Duncan

ACS Earth and Space Chemistry 2017, 1 (6), 361-367

DOI: $10.1021 / a c s e a r t h s p a c e c h e m .7 b 00068$

Long Carbon-Based Chains of Interstellar Medium Can Have a Triplet Ground State. Why Is This Important for Astrochemistry?

loan Bâldea

ACS Earth and Space Chemistry 2019, 3 (5), 863-872

DOI: $10.1021 /$ acsearthspacechem.9b00008

Forsterite Surfaces as Models of Interstellar Core Dust Grains: Computational Study of Carbon Monoxide Adsorption

Lorenzo Zamirri, Marta Corno, Albert Rimola, and Piero Ugliengo

ACS Earth and Space Chemistry 2017, 1 (7), 384-398

DOI: $10.1021 /$ acsearthspacechem.7b00041 


\section{Ammonium Salts as a Source of Small Molecules Observed with High-Resolution Electron- Impact lonization Mass Spectrometry}

Nora Hänni, Sébastien Gasc, Adrian Etter, Markus Schuhmann, Isaac Schroeder, Susanne F. Wampfler, Stefan Schürch, Martin Rubin, and Kathrin Altwegg

The Journal of Physical Chemistry A 2019, 123 (27), 5805-5814

DOI: $\underline{10.1021 / a c s . j p c a .9 b 03534}$

Accessing the Nitromethane $\left(\mathrm{CH}_{3} \mathrm{NO}_{2}\right)$ Potential Energy Surface in Methanol $\left(\mathrm{CH}_{3} \mathrm{OH}\right)-\mathrm{Nitrogen}$ Monoxide (NO) Ices Exposed to lonizing Radiation: An FTIR and PI-ReTOF-MS Investigation

Sándor Góbi, Parker B. Crandall, Pavlo Maksyutenko, Marko Förstel, and Ralf I. Kaiser The Journal of Physical Chemistry A 2018, 122 (9), 2329-2343

DOI: $\underline{10.1021 / a c s . j p c a .7 b 12235}$

\section{Formation of Methane and (Per)Chlorates on Mars}

Svatopluk Civiš, Antonín Knížek, Paul B. Rimmer, Martin Ferus, Petr Kubelík, Markéta Zukalová, Ladislav Kavan, and Elias Chatzitheodoridis ACS Earth and Space Chemistry 2019, 3 (2), 221-232

DOI: $10.1021 /$ acsearthspacechem.8b00104

\section{Pathways to Meteoritic Glycine and Methylamine}

José C. Aponte, Jamie E. Elsila, Daniel P. Glavin, Stefanie N. Milam, Steven B. Charnley, and Jason P. Dworkin

ACS Earth and Space Chemistry 2017, 1 (1), 3-13

DOI: $10.1021 /$ acsearthspacechem.6b00014

\section{Gas Phase Chemistry of Cool Exoplanet Atmospheres: Insight from Laboratory Simulations}

Chao He, Sarah M. Hörst, Nikole K. Lewis, Julianne I. Moses, Eliza M.-R. Kempton, Mark S. Marley, Caroline V. Morley, Jeff A. Valenti, and Véronique Vuitton

ACS Earth and Space Chemistry 2019, 3 (1), 39-50

DOI: $\underline{10.1021 / a c s e a r t h s p a c e c h e m .8 b 00133}$

\section{Condensed-Phase Photochemistry in the Absence of Radiation Chemistry}

Ella Mullikin, Pierce van Mulbregt, Jeniffer Perea, Muhammad Kasule, Jean Huang, Christina Buffo, Jyoti Campbell, Leslie Gates, Helen M. Cumberbatch, Zoe Peeler, Hope Schneider, Julia Lukens, Si Tong Bao, Rhoda Tano-Menka, Subha Baniya, Kendra Cui, Mayla Thompson, Aury Hay, Lily Widdup, Anna Caldwell-Overdier, Justine Huang, Michael C. Boyer, Mahesh Rajappan, Geraldine Echebiri, and Christopher R. Arumainayagam 
Electron-Induced Chemistry: Preliminary Comparative Studies of Hydrogen Production from Water, Methanol, and Diethyl Ether

Demian Marchione and Martin R. S. McCoustra

ACS Earth and Space Chemistry 2017, 1 (6), 310-315

DOI: $10.1021 /$ acsearthspacechem.7b00032

Chemical Composition of Gas-Phase Positive lons during Laboratory Simulations of Titan's Haze Formation

Jennifer L. Berry, Melissa S. Ugelow, Margaret A. Tolbert, and Eleanor C. Browne ACS Earth and Space Chemistry 2019, 3 (2), 202-211

DOI: $\underline{10.1021 / a c s e a r t h s p a c e c h e m .8 b 00139}$

Molecular Evolution of N-Containing Cyclic Compounds in the Parent Body of the Murchison Meteorite

Hiroshi Naraoka, Yohei Yamashita, Mihoko Yamaguchi, and François-Régis Orthous-Daunay ACS Earth and Space Chemistry 2017, 1 (9), 540-550

DOI: 10.1021 /acsearthspacechem.7b00058

Rotational Spectrum of the $\beta$-Cyanovinyl Radical: A Possible Astrophysical N-Heterocycle Precursor

Sommer L. Johansen, Marie-Aline Martin-Drumel, and Kyle N. Crabtree

The Journal of Physical Chemistry A 2019, 123 (24), 5171-5177

DOI: $\underline{10.1021 / a c s . j p c a .9 b 03798}$

Infrared Emission from Photodissociation of Methyl Formate $\left[\mathrm{HC}(\mathrm{O}) \mathrm{OCH}_{3}\right]$ at 248 and $193 \mathrm{~nm}$ : Absence of Roaming Signature

Lucia Lanfri, Yen-Lin Wang, Tien V. Pham, Nghia Trong Nguyen, Maxi Burgos Paci, M. C. Lin, and Yuan-Pern Lee

The Journal of Physical Chemistry A 2019, 123 (29), 6130-6143

DOI: $\underline{10.1021 / a c s . j p c a .9 b 04129}$

Rovibronic Spectroscopy of Sympathetically Cooled ${ }^{40} \mathrm{CaH}^{+}$

Aaron T. Calvin, Smitha Janardan, John Condoluci, Réne Rugango, Eric Pretzsch, Gang Shu, and Kenneth R. Brown

The Journal of Physical Chemistry A 2018, 122 (12), 3177-3181

DOI: $\underline{10.1021 / a c s . j p c a .7 b 12823}$ 
Ionic Species in a Naphthalene Plasma: Understanding Fragmentation Patterns and Growth of PAHs

Martin Alliati, David Donaghy, Xin Tu, and James W Bradley

The Journal of Physical Chemistry A 2019, 123 (10), 2107-2113

DOI: $\underline{10.1021 / a c s . j p c a .9 b 00100}$

High-Resolution Infrared Spectra of the $v_{1}$ Fundamental Bands of Mono-Substituted ${ }^{13} \mathrm{C}$ Propyne Isotopologues

Kirstin D. Doney, Dongfeng Zhao, and Harold Linnartz

The Journal of Physical Chemistry A 2018, 122 (2), 582-589

DOI: $\underline{10.1021 / a c s . j p c a .7 b 10721}$ 